\title{
Handlungsempfehlungen zur Vorbereitung und Implementierung von Spin-Offs und Equity-Carve-Outs
}

\author{
Extrakt des Beitrags "Finanzierungsrelevante Fragestellungen im \\ Kontext von Spin-Offs und Equity-Carve-Outs" von AK \\ Finanzierung der Schmalenbach-Gesellschaft für Betriebswirtschaft \\ e.V.* erschienen in: Schmalenbach IMPULSE, 11. Jg. 2021, Heft 1, \\ https://doi.org/ 10.54585/EMTR2560.
}

Getrieben von technologischen und regulatorischen Umbrüchen, die viele Konzerne zu strategischen Richtungsänderungen zwingen, kann in den jüngsten Jahren ein zunehmender Trend zur (Re-)Fokussierung diversifizierter Konglomerate in Form von Konzernaufteilungen beobachtet werden. Weltweit wurden in den Jahren 2011 bis 2018 jährlich zwischen 150 und 200 Konzernaufteilungen in Form von Equity-Carve-Outs, SplitOffs oder Spin-Offs durchgeführt. Wann bietet sich welche Form der Desinvestition an und welche Problemfelder gibt es aus der Perspektive des Finanzbereichs in der Vorbereitungs- und Implementationsphase einer Konzernaufteilung zu beachten? Der AK Finanzierung der Schmalenbach-Gesellschaft für Betriebswirtschaft e.V. hat dazu eine Fallstudie durchgeführt.

\footnotetext{
AK Finanzierung | Schmalenbach-Gesellschaft für Betriebswirtschaft e.V. | sg@schmalenbach.org

Unter Mitarbeit von Prof. Dr. Hermann Balzer, FH Aachen | Jürgen Binder, Daimler AG | Steffen Diel, SAP SE | Thomas Empelmann, thyssenkrupp AG | Dr. Jochen Gann, Boehringer Ingelheim GmbH | Dr. Jan Haaß, Freudenberg \& Co. KG | Marcel Heinze, Ruhr-Universität Bochum | Jürgen Himmelsbach, TÜV Nord AG | Dr. Christoph Husmann, Encavis AG | Klaus Morgenstern, BASF SE | Prof. Dr. Stephan Paul, Ruhr-Universität Bochum (AK-Leiter) | Peter Schallenberg, Evonik Industries AG | Lorraine Scholle, RuhrUniversität Bochum | Kai Schrickel, Robert Bosch GmbH | Dr. Roger Schweitzer, Bertelsmann AG | Marco Swoboda, Henkel KGaA | Dr. Verena Volpert, ehemals E.ON SE. Der Arbeitskreis ist Marcel Heinze und Lorraine Scholle zu besonderem Dank verpflichtet, da sie die Diskussionen in den Sitzungen vorbereitet und den Entwurf dieses Manuskripts erstellt haben.
} 


\section{Schritt 1: Spin-Off oder Equity-Carve-Out?}

Bei einem Spin-Off (SO) werden die Anteile des aufnehmenden Rechtsträgers nach der Abspaltung zum öffentlichen Handel zugelassen (OneStep-Spin-Off). Wenn der übertragende Rechtsträger zu diesem Zeitpunkt noch über Anteile an dem aufnehmenden Rechtsträger verfügt, hat er die Möglichkeit, diese über den öffentlichen Handel zu veräußern und entsprechend Liquidität zu generieren (Two-Step-Spin-Off).

Im Gegensatz zu einem SO wird im Rahmen eines Equity-Carve-Outs (ECO) die Beteiligung an einer bestehenden oder neu gegründeten Gesellschaft über ein Initial Public Offering (IPO) an externe Investoren verkauft. Das heißt, es fließt unmittelbar Liquidität zu.

Bei der Entscheidung zwischen einem SO oder einem ECO hat das Management mindestens drei Fragen zu klären:

1. Wie hoch ist der Liquiditätsbedarf des ehemaligen Mutterunternehmens?

2. Was sind die unternehmensspezifischen Faktoren des potenziellen neuen Unternehmens?

3. Sollten die Unternehmensteile vollständig separiert werden oder über gegenseitige Beteiligungsverhältnisse miteinander verbunden bleiben?

\section{Schritt 2: Neuorganisation der Konzernstruktur}

Vor Aufspaltung eines Konzerns ist dieser zunächst so zu restrukturieren, dass die operativen Geschäfte überschneidungsfrei den Tochter- bzw. Mutterunternehmen zugeordnet werden. Die Komplexität der $\mathrm{Re}-$ organisation ergibt sich aus der Komplexität der Ausgangsstruktur.

Im Zuge der Neuorganisation der Konzernstruktur ist die Form der Desinvestition bereits mitzudenken, denn hieraus ergeben sich weitreichende Konsequenzen für:

- die Möglichkeiten zur Steuerung der Zielkapitalstruktur,

- die Wahrscheinlichkeit des Auftretens von Interessenkonflikten bei der Ressourcenallokation,

- die Anforderungen der Banken bei den Verhandlungen über eine externe Finanzierungsstruktur sowie

- die mittel- bis langfristige Kapitalmarktperformance der involvierten Unternehmensteile.

\section{Schritt 3: Definition Zielrating und Ratingprozesse}

Die Definition des Zielratings eines Unternehmensteils zählt zu den Kernkompetenzen der Finanzorganisation. Aus dem Zielrating leitet sich die Zielkapitalstruktur ab. Und diese ist bestimmend für die Verschuldungsrestrukturierung. 
Das Risikoprofil der jeweiligen Unternehmensteile setzt den Rahmen für das Zielrating. Bei der Beurteilung der mit der Transaktion verbundenen Konsequenzen für die finanziellen Kennziffern sowohl der Mutter-als auch der Tochterunternehmen ist die frühzeitige Einbindung von Ratingagenturen notwendig. Um die Ratingprozesse effizient durchzuführen, muss das Management in kurzer Zeit zuverlässige Informationen - wie separate Abschlüsse, Combined Financial Statements und BusinessPläne - zur Verfügung stellen.

\section{Schritt 4: Definition und Implementierung der Zielkapitalstruktur}

Das Geschäftsrisiko und das avisierte Zielrating bilden die Leitplanken für die Zielkapitalstruktur der aus der Transaktion erwachsenen Unternehmen. Abhängig von der gewählten Transaktionsstruktur stellen Veräußerungserlöse aus dem Börsengang des Tochterunternehmens ein Instrument zur Steuerung der Zielkapitalstruktur dar. Es ergeben sich außerdem unterschiedliche Implikationen für die Reallokation des bestehenden Fremdkapitals sowie für den Aufbau einer externen Finanzierungsstruktur für das Tochterunternehmen.

Der AK Finanzierung beleuchtet in seiner jüngsten Studie mit beteiligten Finanzverantwortlichen in fünf bereits durchgeführten bzw. begonnenen Konzernaufteilungen typische Abläufe innerhalb dieser Prozessschritte. Die daraus hervorgehenden Problemfelder sowie „Best Practices“ werden in Handlungsempfehlungen überführt, die durch den bisher einmaligen Einblick in die Unternehmenspraxis zur Berücksichtigung bei zukünftigen Konzernaufteilungen nützlich sein können. 